\section{Molecular Formula and Degree of Unsaturation}

A relation stated without proof or reference by Soffer ${ }^{1}$ deserves to be better known. Ideas more or less equivalent to it seem to be widely known to organic chemists, but workers in related fields may fird the result useful when doaling with organic compounds. This communication presents a proof, together with some remarks on interpretation and use (supplementary to Soffer's remarks).

For a start, consider a molecule the molecular (numerical) formula of which is known. and the atoms of which can safoly be regarded as covalent with predictable valencies. Thus we can calculate the sum $V$ of the valencies of all the atoms. The number of bor.ds in the structure must be $\frac{1}{2} V$ because each bord satisfies two valencies.

But if there are $N$ atoms then as fow as $N \sim 1$ bonds are sufficient to link them together-one bor.d would serve to link two atoms, a secord bond would allow a third atom to be added, and so on.

If this were fewer than $\frac{1}{2} V$, then each of the extra bonds must link atoms which are already linked either directly or indirectly in the molecule. If indirectly, then such an extra bord completes a rirg; if directly, it gives us a double bond (or increases the order of a multiple bord). A.lways we get what Soffer calls a "cyclic element of structure". (A triple bond is to be counted as two cyclic elements.) Thus the number of these cyclic elements is predicted as the number of 'surplus' bonds:

$$
p \text { (say) }=\frac{1}{2} V-N+1
$$

Also $2 \rho$ is the number of added hydrogens required to saturate the molecule (eliminating all bond-duplications and rings): because each pair of hydrogens eliminates one of the 'surplus' bonds.

(1) We seo that two molocules have the same p-value whenever their molecular formulæ are identical (isomeric): or when the formulæ differ only by multiples of $\mathrm{CH}_{2}, \mathrm{NH}$ and/or $O$, because these combinations contribute zero to $\left(\frac{1}{2} V-N\right)$.

(2) Equation (1) may be written:

$$
\rho=\frac{1}{2} \Sigma v n_{v}-\Sigma n_{v}+1
$$

where $n_{v}$ is the number of atoms of valency $v$, and rearranged to give Soffer's form:

$$
\rho=1+\frac{1}{2} \Sigma n_{v}(v-2)
$$

(that is, $\rho=1+\frac{1}{2}\left(2 n_{4}+n_{3}-n_{1}\right)$ if no higher valencies are present). This gives a quicker calculation if it can be remembered.

(3) Soffer's definition, "in counting the number of cyclic structures in a polycyclic system no cyclic structure is counted if it is made up entirely of elements of other cyclic structures which themselves have been counted", can give ambiguous results in some cases. Thus tetradecahydroanthracere, $\mathrm{C}_{14} \mathrm{H}_{24}$, gives $p=3$ and has indeed three six-membered rings:

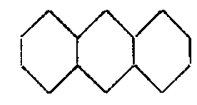

but could be counted as having only two rings, the middle one and the 'outside'. In such a case. we must say that $p$ predicts the number of bonds that must be cut to eliminate all the cyclic structures. (For formally planar structures it is sufficient to count non-overlapping rings.)

(4) The equation holds if the true structure has the atoms in their predicted covalencies. or if an acceptable model of the structure has this property. Thus aromatic nuclei may be considered as having Kekulé structures. as Soffer apparently irtends when he cites benzylpenicillin and strychnine. (Alternatively we may arbitrarily call each aromatic $(\pi)$ electron-pair a 'cyclic structure'.) A benzene ring is a possible explanation of four units of a calculated unsaturation.
Similar considerations apply to the groups - NO and $-\mathrm{NO}_{2}$ (count one cycle each); but substituted ammonium is more d'fficult to deal with as it appears 'supersaturated' until we calculate again using valency five for the nitrogen.

(5) The equation may be generalizod to a sot of atoms other than a single complete molecule. By adding equations for $M$ separate molecules we obtain:

$$
\rho=\frac{1}{2} V-N+M \text { or } \rho-M=\frac{1}{2} V-N
$$

where $N, V$, $\rho$ are the total number of atoms. valencies and cyclic elements in the $M$ molecules. Hore $M$ roplaces the I of equation (1) (or (2), sim larly). On the other hand, we must subtract $\frac{1}{2}$ from the same figure for each valency either not satisfied (as in a free radical), or not satisfied within the set of atoms (such as the monomeric unit of a dimer or polymer, or 'structural units' of coal').

\section{David A. Laws}

\section{Dominion Physical Laboratory,}

$$
\text { Lower Hutt, }
$$

Now Zealand.

1 Soffer, M. D., Science, 127, 880 (1958).

${ }^{2}$ van Krevelen, D. W., Coal, 436 (Amsterdam, Elsevier, 1961).

\section{Near Ultra-violet Absorption Spectra of $0-, m-$ and $p$-Fluorophenol Vapour}

THE absorption spectra of $o-, m$-, and $p$-fluorophenol in vapour phase have been examined with a Hilger $E_{1}$ large quartz spectrograph. A number of plates were taken with different cell lengths and at temperatures ranging from $26^{\circ} \mathrm{C}$ to $60^{\circ} \mathrm{C}$; while the temperature of the reservoir containing the substance varied from $-15^{\circ} \mathrm{C}$ to $60^{\circ} \mathrm{C}$. The bands in each case appear well defined with the cell of length $70 \mathrm{~cm}$ maintained at temperature $26^{\circ} \mathrm{C}$. A hydrogen lamp was used as the source of continuum for the ultra-violet region. Ilford special rapid $N .40$ plates were used to photograph the spectra in about half an hour.

Nearly 300 bands have been photographed in each case. The intense bands are double headed, similar to those observed in the case of phenol. The moan doublet separation in all the three spectra is found to be of the order of $10 \mathrm{~cm}^{-1}$.

o-Fluorophenol: This molecule belongs to symmetry class $C s$ to a first approximation and its absorption spectrum is due to the electronic transition ${ }^{1} A^{\prime} \leftarrow^{1} A^{\prime}$. The most intense band at $36,802 \mathrm{~cm}^{-1}$ has been chosen as the $(0,0)$ band, and the entire spectrum analysed in terms of the frequencies $239,415,566$ and $766 \mathrm{~cm}^{-1}$ in the ground state and $291,506,718,945,1,040$ and 1,128 $\mathrm{cm}^{-1}$ in the excited state. The ground state frequencies correspond fairly closely to the known Raman frequencies.

m-Fluorophenol: The meta-derivative of the molecule can also be taken to belong to the symmetry class $C_{s}$ and the bands may be attributed to the electronic transition ${ }^{1} A^{\prime} \longleftarrow^{1} A^{\prime}$ which is an allowed transition. The intense band at $36.620 \mathrm{~cm}^{-1}$, which is observed with still greater intensity at low temperature. is taken to be the $(0,0)$ band. The entire spectrum is analysed in terms of two ground state frequencies, 427 and $741 \mathrm{~cm}^{-1}$, and six excited state frequencies, 146, 206, 254, 713, 976 and $1,175 \mathrm{~cm}^{-1}$.

p-Fluorophenol: The para-derivative belongs to $C_{2 v}$ symmetry class approximately, and the observed spectrum involves the electronic transition ${ }^{1} A_{1} \leftarrow^{1} B_{1}$, which is allowed with the transition moment lying in the molecular plane and perpendicular to $\mathrm{C}-\mathrm{OH}$ bond. The most intense band at $35.132 \mathrm{~cm}^{-1}$, which appears with increased intensity at lower temperatures, has been taken to be the $(0,0)$ band. The bands have been analysed in terms of three ground state frequencies 251.466 and $865 \mathrm{~cm}^{-1}$, and seven excited state frequencies. $356,413,569,838,875$, 1,205 and $1,273 \mathrm{~cm}^{-1}$. Satisfactory correspondence of the 